\title{
Diabetic retinopathy screening during the coronavirus disease 2019 pandemic
}

\author{
Kendrick C. Shih $\circledast^{1}$ - Alfred S. K. Kwong ${ }^{2}$ - Jenny H. L. Wang ${ }^{2}$ - Jasper K. W. Wong $\circledast^{1} \cdot$ Welchie W. K. Ko ${ }^{2}$. \\ Jimmy S. M. Lai ${ }^{1} \cdot$ Jonathan C. H. Chan (1) ${ }^{1}$
}

Received: 13 April 2020 / Revised: 22 April 2020 / Accepted: 22 April 2020 / Published online: 4 May 2020

(c) The Royal College of Ophthalmologists 2020

\section{To the Editor:}

The current focus on the coronavirus disease 2019 (COVID-19) pandemic have invariably led to some compromises in medical care [1] and the curtailing of health services, including screening programs. This is reflected by the recent recommendation of the Royal College of Ophthalmologists on postponing routine diabetic retinopathy (DR) screening of patients with diabetes mellitus [https:// rcophth.ac.uk/2020/03/covid-19-update-and-resources-forophthalmologists/]. Although it helps prevent visual loss [2], we understand that postponing DR screening may be necessary when facing severe shortages in staff, personal protective equipment, and escalating new infections. However, in areas with effective infection control and low community transmission, we believe it can be continued safely, especially when incorporating advances in telemedicine. Such is the case with DR screening in Hong Kong. Our department had previously played a leading role in researches [3] which led to the establishment of universal DR screening in Hong Kong since 2014. The current program involves scheduling patients with diabetes for DR screening every 1-2 years (depending on coexisting risk factors). During screening, patients' visual acuity and digital fundal photos are uploaded to a secure, central server. The uploaded images are graded remotely at secure workstations according to a standardized protocol [4]. Patients with sight-threatening stages of DR are referred to ophthalmologists for further management.

Jonathan C. H. Chan

jonochan@hku.hk

1 Department of Ophthalmology, University of Hong Kong, Hong Kong, Hong Kong

2 Department of Family Medicine and Primary Healthcare, Hong Kong West Cluster, Hospital Authority, Hong Kong, Hong Kong
The Department of Family Medicine and Primary Healthcare (FM\&PHC) of Hong Kong West Cluster, as with other FM\&PHC departments throughout Hong Kong, have continued DR screening throughout this period. As the causative agent for COVID-19 can be present in ocular secretions [5], additional measures have been adopted to reduce transmission risk, including:

(1) Screening of all visitors for: body temperature for fever, history of respiratory symptoms, recent overseas travel, and possible contact or exposure to COVID-19 cases. Those screened positive had their screening postponed (if patients) and referred to designated hospitals for suspected cases.

(2) Universal face-covering with surgical or N95 masks by staff and all visitors. Patients arriving without surgical masks were provided one.

(3) Protective plastic shields installed on all slit-lamps, which are used during eye examinations.

(4) Number of bookings reduced to avoid overcrowding within the clinics.

As bookings were intentionally reduced since February 2020 , the number of attendances decreased compared with the same period in 2019 (Table 1). Comparing the first quarter of 2019 and 2020, bookings and attendance decreased by $24.0 \%$ and $27.6 \%$, respectively, with a small rise in default rate from $15.1 \%$ to $19.1 \%$ (Fig. 1). The

Table 1 Number of bookings, attendances, and defaults for January, February, and March of 2019 and 2020.

\begin{tabular}{lllllll}
\hline & Jan-19 & Feb-19 & Mar-19 & Jan-20 & Feb-20 & Mar-20 \\
\hline Bookings & 777 & 567 & 819 & 550 & 489 & 605 \\
Attendances & 663 & 477 & 696 & 469 & 368 & 493 \\
Defaulted & 114 & 90 & 123 & 81 & 121 & 112 \\
Default rate \% & 14.7 & 15.9 & 15.0 & 14.7 & 24.7 & 18.5 \\
\hline
\end{tabular}




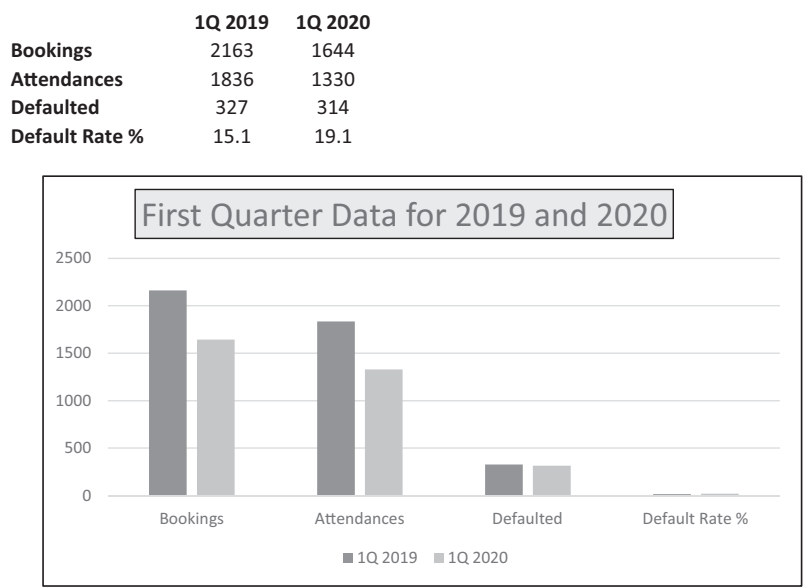

Fig. 1 First quarter data for 2019 and 2020. Total bookings, attendances, and defaults for the first quarter of 2019 (1Q 2019) and 2020 (1Q 2020).

default rate was especially high for February 2020, when there was widespread fear of acquiring COVID-19 by many patients at the time. It has since improved slightly for March 2020. As of 12th April 2020, there have been no reported cases of COVID-19 infection among our clinical staff, nor any patients who underwent DR screening during this period.

We believe it is safe and worthwhile, when resources and infection control measures are available, to continue with
DR screening, especially for patients with poor diabetic control, in view of the likely protracted course of the current COVID-19 outbreak.

\section{Compliance with ethical standards}

Conflict of interest The authors declare that they have no conflict of interest.

Publisher's note Springer Nature remains neutral with regard to jurisdictional claims in published maps and institutional affiliations.

\section{References}

1. Abbasi K. The scandals of covid-19. Editorial. BMJ. 2020;369: m1434.

2. Early photocoagulation for diabetic retinopathy. ETDRS report number 9. Early Treatment Diabetic Retinopathy Study Research Group. Ophthalmology. 1991;98:766-85.

3. Lian JX, McGhee SM, Gangwani RA, Hedley AJ, Lam CL, Yap MK, et al. Screening for diabetic retinopathy with or without a copayment in a randomized controlled trial: influence of the inverse care law. Ophthalmology. 2013;120:1247-53. https://doi.org/10. 1016/j.ophtha.2012.11.024

4. Gangwani RA, Lian JX, McGhee SM, Wong D, Li KK. Diabetic retinopathy screening: global and local perspective. Hong Kong Med J. 2016;22:486-95.

5. Xia J, Tong J, Liu M, Shen Y, Guo D. Evaluation of coronavirus in tears and conjunctival secretions of patients with SARS-CoV-2 infection. J Med Virol. 2020. https://doi.org/10.1002/jmv.25725. 